\title{
Alternative Approach to Animal Testing and Cell Cultures, According to European Laws
}

doi:10.14573/altex.1706231

On September 29-30, 2016, the $8^{\text {th }}$ edition of the training course on "Alternative Approach to Animal Testing and Cell Cultures, According to European Laws", was held in Genoa, chaired by Prof. Anna Maria Bassi and organized and hosted by the team of Laboratory Analysis and Research in Physiopathology (LARF), Department of Experimental Medicine, University of Genoa, Italy. The course was focused on practical work and demonstration/lessons by specialist(s) aiming to supply basic knowledge or improve existing knowledge on alternative methods. Each edition of the course provides an update on innovative in vitro models with a particular emphasis on 3D models. Substantial practical time is included to allow participants to gain hands-on experience. Twenty-four participants, from experts of in vitro methods to undergraduates, from all over Italy took part.

The course was opened by Prof. Giovanni Murialdo and Prof. Adriana Voci, coordinators of the degree courses in Medicine and Surgery, and Biological Sciences, respectively, at the University of Genoa. Both stressed the need for such events to ensure that research in Italy for the study of risks to human and animal health following exposure to chemical compounds (drugs, pollutants, food additives, etc.) is in step with new technologies.

Prof. Rosagemma Ciliberti (DISSAL, University of Genoa) and Susanna Penco (LARF-DIMES, University of Genoa) highlighted ethical issues of animal-based research. According to Italian Law 413/1993 on "Rules on conscientious objection to animal experimentation", physicians, researchers, students and healthcare providers can choose not to take part in activities that involve animal experimentation. Conscientious objection to animal experimentation arose from the acknowledgement of animals as conscious beings.

Dr Chiara Scanarotti (LARF-DIMES, University of Genoa) gave an update on the development and validation of several well-standardized and reproducible alternative methods for toxicity testing of chemical compounds, underlining how the use of human instead of animal cells improves the accuracy and relevance of the in vitro tests. The lecture highlighted 3D models, spheroids, body-on-a-chip and the latest advancements in stem cell research.

Dr Costanza Rovida (CAAT Europe - University of Konstanz, Germany) presented a lecture on the implementation of alternative methods. Full replacement of in vivo tests is a lengthy process as they are mandatory for the registration and risk assessment of chemicals (drugs, additives, etc.) and the use of alternative methods requires formal validation before acceptance. To date, in vitro tests are accepted for some endpoints, such as skin/eye irritation and partially for skin sensitization. Some non-validated alternative approaches, such as read-across and weight-of-evidence approaches, are also accepted in the EU when sufficiently justified. The OECD launched a program on the development of Adverse Outcome Pathways (AOP) in 2012. AOP is an analytical construct that starts from a mechanistic/ cellular/in vitro sub-profiling to reach the effects observed in populations, and describes sequential events at different levels of biological organization that lead to adverse health or ecotoxicological effects.

Dr Stefania Vernazza (LARF-DIMES, University of Genoa) provided an overview on in vitro $3 \mathrm{D}$ alternative methods for evaluation of the human risk related to exposure to chemical compounds. International organizations, such as ECVAM and OECD, cooperate on the development of regulations on reducing the use of laboratory animals through the validation of in vitro toxicity tests. Some OECD test guidelines (439, 431 and 492) accept data from in vitro 3D models to classify a substance as irritant/corrosive for skin or eye without the need to perform animal tests. 3D tissues for these applications are commercially available.

Prof. Anna Maria Bassi (LARF-DIMES, University of Genoa) explained the concept of a reliable predictive toxicology. Most of the failures in drug discovery are a result of the low predictivity of preclinical animal models for human diseases such as autism syndrome or asthma. There is the pressing need for new toxicity testing models based on human biology. In this regard, Dr Bassi spotlighted the development of a mini-brain by Thomas Hartung's team (CAAT, Johns Hopkins University) to improve research and drug development for neurodegenerative diseases. Mini-brains derive from patient cells and so can reproduce epigenetic interactions. The second part of the lecture was focused on AOPs, which provide a comprehensive knowledge of human disease and link chemical properties of a toxicant, its macro-molecular interactions, cellular/organ/organism responses and adverse outcomes in the population. This complex of data is critical to identify exposure biomarkers and toxicity pathways. The OECD's AOP website invites project proposals, offers stakeholder summaries, proposal templates and guidance on developing AOPs. The AOP program foresees a biannual update for new project proposals and new information on ongoing projects.

Dr Giorgio Mattei (Research Centre "E. Piaggio", University of Pisa, Italy) presented an update on innovative technologies for dynamic in vitro 3D models. He explained the characteristics of biomimetic in vitro models resulting from combinations of cell culture systems, advanced materials design, tissue engi- 
neering strategies, scaffold fabrication and non-invasive realtime monitoring technologies in multi-chamber bioreactors.

Dr Paola Visconti (Biological Bank, IRCSS University Hospital San Martino-IST, Genoa, Italy) gave an overview on the organization, services and Italian/European network of biobanks. The Biological Bank offers quality controlled human and animal cell lines, including detection and eradication of mycoplasma, and the possibility to deposit cell lines and hybridomas to make them available for the scientific community or for patent purposes. Human cell lines are identified by applying the technique of Multiplex short tandem repeat (STR) profiling. Dr Visconti showed how the Biological Bank has contributed to the definition of the OECD Guidelines for the management of Biological Resource Centers (CRB) and participates actively in the European network of cell banks (BBMRI project) and the national network of biobanks of Alliance Against Cancer.

Dr Valery Shevchenko (Biopredict International, Saint Grégoire, France) outlined applications of the human cell line HepaRG $^{\mathbf{T M}}$, which are terminally differentiated hepatic cells derived from a human hepatic progenitor cell line that retains many characteristics of primary human hepatocytes and can undergo differentiation and transdifferentiation stages. HepaRG ${ }^{\mathrm{TM}}$ cells exhibit a transcriptome-stable hepatocyte phenotype, morphology and specific functions, such as sugar, lipid, nitrogen, bile acid metabolism, expression of key metabolic enzymes, all nuclear receptors, drug transporters and high P450 activity. HepaRG $^{\mathrm{TM}}$ spheroids can be produced by hanging drops, AggreWell ${ }^{\mathrm{TM}}$, ultra-low attachment, stirred perfusion reactor, etc. An innovative model is the co-culture of HepaRG ${ }^{\text {TM }}$ and human stem cells for development of a human liver organoid to investigate drug-induced fibrosis.

Dr Carlo Lusso (Euroclone - Lonza, Pero, Milan, Italy) explained the criteria for choosing between primary cells and cell lines for research. Primary cells from normal and diseased donors, which are offered by the company, show tissue char- acteristics similar to in vivo conditions, however, these have a limited lifespan in comparison to cell lines.

An extensive practical session allowed participants to try out some technologies. The training blocks were introduced by company specialists, with the support of the LARF team, Dr Chiara Scanarotti, Stefania Vernazza and Sara Tirendi. Dr Tomaso Sbrana (IVTech, Massarosa, Lucca, Italy) provided the opportunity for hands-on experience with 3D dynamic in vitro models in bioreactors. Dr Shevchenko (Biopredic International) provided the opportunity to observe HepaRG ${ }^{\text {TM }}$ hepatic stem cell morphology, spheroid formation, HepaRG ${ }^{\text {TM }}$ hepatocyte polarization, bile canaliculi (BC) presence, and functional MRP2 (multi-drug resistance protein 2).

Fruitful discussions during the hands-on sessions and in a final roundtable set the stage to create a network for scientific exchange of skills among experts and trainees. The evaluation questionnaires, completed at the end of the modules, resulted in a high level of satisfaction by all participants, who requested that other courses be scheduled for acquisition of basic techniques or for improvement of existing expertise on alternative methods.

We thank Dr Helena Kandarova, Dr Silvia Letasiova, Dr Giorgio Mattei, Dr Tommaso Sbrana, Dr Valery Shevchenko, and Dr Carlo Lusso for their invaluable contribution to the success of the course.

Chiara Scanarotti ${ }^{1}$, Costanza Rovida ${ }^{2}$, Susanna Penco ${ }^{1}$, Stefania Vernazza ${ }^{1}$, Sara Tirendi ${ }^{1}$, Rosagemma Ciliberti ${ }^{3}$ and Anna Maria Bassi ${ }^{1}$

${ }^{1}$ Department of Experimental Medicine (DIMES), University of Genova, Italy; ${ }^{2}$ CAAT-Europe, University of Konstanz, Konstanz, Germany; ${ }^{3}$ Department and of Health Sciences (DISSAL), University of Genova, Italy 